\title{
A NEW METHOD FOR WARING THEOREMS WITH POLYNOMIAL SUMMANDS*
}

\author{
BY \\ L. E. DICKSON
}

1. Part I of this paper is self-contained and presupposes only the rudiments of elementary theory of numbers. The method employs a pair of polynomials $p(x)$ and $q(x)$ of degree $n$, each uniquely determined by the other, such that there exists an identity which expresses $I q(s)$ as a sum of $m$ values of $p\left(x^{2}\right)$, where $I$ is an integer and $s$ is a sum of four squares. Then a Waring theorem for $q(x)$ yields one for $p\left(x^{2}\right)$. For, if every (large) integer is a sum of $v$ values of $q(x)$, then every (large) multiple of $I$ is a sum of $v m$ values of $p\left(x^{2}\right)$. From the last result we readily find how many values of $p\left(x^{2}\right)$ suffice for all integers.

Apart from the special case in which $q(x)$ is a power of $x$, there is no hint in the literature of this instantaneous deduction of a Waring theorem for an even polynomial of degree $2 n$ from a known Waring theorem for a polynomial of degree $n$. On the contrary, Maillet resorted to an extensive proof for the case $n=2$.

We feel justified in perfecting the theory of sums of four values of a quadratic function $q(x)$ in view of the resulting theorems for certain polynomials of degrees 4,8 , etc.

Since we seek Waring theorems holding for all positive integers (or with all exceptions listed), we are not content with theorems holding for all sufficiently large integers and certainly not with the asymptotic results much in vogue.

PART I. WARING THEOREMS FOR POLYNOMIALS OF DEGREES 2 AND 4

2. Using the abbreviation $s=a^{2}+b^{2}+c^{2}+d^{2}$, we have the identities

$$
6 s \equiv \sum_{12}(a \pm b)^{2}, 6 s^{2} \equiv \sum_{12}(a \pm b)^{4},
$$

in which the summands are the powers of

$$
a \pm b, a \pm c, a \pm d, b \pm c, b \pm d, c \pm d .
$$

Write

$$
f(x)=u x^{4}+v x^{2}, q(x)=u x^{2}+v x .
$$

* Presented to the Society, April 7, 1934; received by the editors June 29, 1934. 
Hence we have the following identity* in $a, b, c, d, u, v$ :

$$
6 q(s) \equiv \sum_{12} f(a \pm b) \text {. }
$$

First, take $u=v=1 / 2$. Then $q(x)=x(x+1) / 2$ is called a triangular number. It is known $\dagger$ that every positive integer $n$ is a sum of three triangular numbers $q\left(s_{i}\right)$ with $s_{i} \geqq 0$. But each such integer $s_{i}$ is a sum of four squares. Hence (4) used three times shows that $6 n$ is a sum of 36 values of $f(x)=$ $\left(x^{4}+x^{2}\right) / 2$ for integers $x$. Every positive integer $p$ is of the form $6 n+r$, $0 \leqq r \leqq 5$, while $r=r f(1)$, whence $p$ is a sum of 41 values of $f(x)$.

We can reduce 41 to 38 as follows. The numbers $f(0)=0, f(1)=1, f(2)=10$, $f(3)=45$ are congruent modulo 6 to $0,1,4,3$. Also, $2 f(1)=2, f(1)+f(2) \equiv 5$ ( $\bmod 6$ ). Hence if $M$ is any integer, we can find two integers $a, b$, each $\geqq 0$, such that

$$
f(a)+f(b) \equiv M(\bmod 6), f(a)+f(b) \leqq 45 .
$$

Thus if $M \geqq 45, M-f(a)-f(b)$ is a multiple $\geqq 0$ of 6 and hence is a sum of 36 values of $f(x)$, whence $M$ is a sum of 38 such values. But $10 x+y=x f(2)+y f(1)$ is a sum of $x+y$ values of $f(x)$, whence every integer $<100$ is a sum of fewer than 20 values.

THEOREM 1. Every positive integer is a sum of 38 values of $\left(x^{4}+x^{2}\right) / 2$.

3. Second, take $u=1 / 2, v=-1 / 2$. Then $q(x)=\left(x^{2}-x\right) / 2$ becomes $\left(y^{2}+y\right) / 2$ for $y=x-1$. We may discard the negative value -1 of $y$ corresponding to $x=0$ since $q(x)=0$ also when $x=1$, which corresponds to $y=0$. The fact that every integer $N \geqq 0$ is a sum of three triangular numbers therefore implies that $N$ is a sum $\ddagger$ of three values of $q(x)$ for integers $x \geqq 0$. Thus (4) implies that every positive multiple of 6 is a sum of 36 values of $f(x)=\left(x^{4}-x^{2}\right) / 2$ for integers $x$. Conversely, any sum of values of $f(x)$ is a multiple of 6 . In fact, if $x$ is any integer, $x^{4}-x^{2}$ is a multiple of 4 and of 3 .

THEOREM 2. Every positive integer is a sum of 36 (always positive integral) values of $\left(x^{4}-x^{2}\right) / 12$.

4. Maillet $\S$ investigated positive integers $A$ which are sums of four values of

* Also when we add $c$ to $f$ and $2 c$ to $g$. The modifications of the later theory are evident.

$\dagger$ Since $8 n+3$ is a sum of three squares, each necessarily an odd square $(2 x+1)^{2}$, where $x \geqq 0$. Hence $n=\Sigma q(x)$.

$\ddagger$ This $q(x)$ and $\frac{1}{2} x(x+1)$ are the only functions $q(x)=u x^{2}+v x$ such that every positive integer is a sum of three values of $q(x)$ and such that $q(x)$ is an integer $\geqq 0$ for every integer $x \geqq 0$.

$\S$ Bulletin de la Société Mathématique de France, vol. 23 (1895), pp. 40-49. He did not find the actual limit (12) for $A$. 


$$
q(x)=\left(m x^{2}+n x\right) / 2, m>0 .
$$

If we can find positive odd integers $k$ and $t$ such that

$$
\begin{gathered}
(3 k-2)^{1 / 2}-1<t<(4 k)^{1 / 2}, \\
A=m k / 2+n t / 2,
\end{gathered}
$$

then by Cauchy's lemma there exist four integers $\geqq 0$ whose sum is $t$ and the sum of whose squares is $k$, whence by (5) and (7), $A$ is a sum of four values of $q(x)$ for integers $x \geqq 0$. Write

$$
r^{2}=n^{2}+2 A m, w^{2}=24 A m+12 m n-8 m^{2}+9 n^{2} .
$$

Let $A \geqq m / 2$, whence $w^{2} \geqq(2 m+3 n)^{2}$. Elimination of $k$ between (6) and (7) gives

$$
L \equiv \frac{-2 m-3 n+w}{2 m}<t<G \equiv \frac{-2 n+2 r}{m}, L \geqq 0 .
$$

Let $m$ and $n$ be relatively prime odd integers. If $G>L+2 m$, there are $m$ consecutive positive odd integers $t_{1}, \cdots, t_{m}$ between $L$ and $G$ in (9). If $2 A-n t_{i} \equiv 2 A-n t_{i}(\bmod m)$, then $t_{i}$ and $t_{j}$ are congruent modulo $m$ and hence modulo $2 m$. Since their difference is numerically $<2 m$, they are equal. Hence there is a positive odd integer $t$ satisfying both $(9)$ and $2 A-n t \equiv 0(\bmod m)$. Then $k=(2 A-n t) / m$ is an odd integer satisfying (7). This $k$ is positive since $2 A \geqq n G(>n t)$, which follows by eliminating $A$ by $\left(8_{1}\right)$ and using $(r-n)^{2} \geqq 0$.

Conversely, when $k$ and $t$ are positive integers, (7) and (9) imply (6) if $m t+2 n \geqq 0$ (trivial if $n \geqq 0$ ) and hence if $m L+2 n \geqq 0$, viz., $w \geqq 2 m-n$. The latter follows from its square and hence from

$$
\left.6 m A \geqq 3 m^{2}-4 m n-2 n^{2} \quad \text { (if } n<0\right) .
$$

The condition $G-L>2 m$ is equivalent to

$$
4 r>w+T, T=n-2 m+4 m^{2} .
$$

This follows from its square: $16 r^{2}-w^{2}-T^{2}>2 w T$. The latter follows from its square (11) if its left member is $\geqq 0$, which is true when

$$
8 A m \geqq T^{2}-7 n^{2}+12 m n-8 m^{2} .
$$

Write $w^{2}=24 A m+H$. Then

$$
\begin{aligned}
(8 A m+J)^{2} & =\left(16 r^{2}-w^{2}-T^{2}\right)^{2}>(2 w T)^{2}=4 T^{2}(24 A m+H), \\
H & =12 m n-8 m^{2}+9 n^{2}, J=8 m^{2}-12 m n+7 n^{2}-T^{2} .
\end{aligned}
$$

In the inequality transpose the terms with $A$ to the left and complete the square on $A$. Hence 


$$
\begin{gathered}
8 A m>6 T^{2}-J+2 T P, P^{2}=H-3 J+9 T^{2}, \\
P^{2}=16 m^{2}\left(1+6 n-12 m+12 m^{2}\right) .
\end{gathered}
$$

Conversely, if $P \geqq 0, T \geqq 0$, (12) implies (11).

THEOREM 3. If $m$ and $n$ are relatively prime odd integers and $m>0, P \geqq 0$,

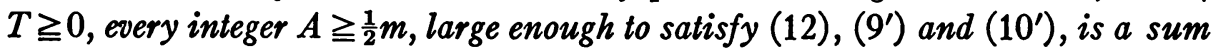
of four values of (5) for integers $x \geqq 0$.

5. Hence (4) used four times shows that $6 A$ is a sum of 48 values of $f(x)=\left(m x^{4}+n x^{2}\right) / 2$. Let $D$ denote the g. c. d. of 6 and $f(1)$. Let $g$ be any given multiple of $D$. Then $f(1) y+6 z=g$ is solvable in integers $y, z$, with $0 \leqq y \leqq 5$. Thus $6 N+g=6(N+z)+y f(1)$. Hence every sufficiently large multiple of $D$ is a sum of $48+y \leqq 53$ values of $f(x)$. This is equivalent to the more complicated Theorem V of Maillet, who proved it by an extensive argument based on Cauchy's lemma.

It is a new result that we may replace 53 by 50 . Write

$$
m=2 M+1, n=2 N-1,
$$

where $M$ and $N$ are integers. Then

$$
f(0)=0, f(1)=M+N, f(2) \equiv 4(M+N), f(3) \equiv 3(M+N) \quad(\bmod 6) .
$$

These with $2 f(1)$ and $f(1)+f(2)$ evidently form a complete set of residues modulo 6 if $M+N$ is prime to 6 . Let $G$ be the largest of the six numbers just used. Let $I$ be any integer $\geqq G$. Hence there exist integers $a, b$, each $\geqq 0$, such that $f(a)+f(b)$ is $\leqq G$ and is $\equiv I(\bmod 6)$. Thus $I-f(a)-f(b)$ is a positive multiple $6 A$ of 6 . If $A$ is large enough to satisfy (12), $6 A$ is a sum of 48 values of $f(x)$.

Next, if $M+N$ is even and prime to 3, we see that $f(0), f(1)$ and $2 f(1)$ are congruent modulo 6 to $0,2,4$ in some order. Thus all large even integers are sums of 50 values of $f(x)$, which is always even.

Next, if $M+N$ is an odd multiple of $3, f(1) \equiv 3(\bmod 6)$. If $I$ is a multiple of 3 , one of $I, I-f(1)$ is a multiple of 6 .

THEOREM 4. Let $m$ and $n$ be relatively prime odd integers, $m>0$. Let $D$ denote the g.c.d. of 6 and $(m+n) / 2$. Then every sufficiently large multiple $I$ of $D$ is a sum of 50 values of $f(x)=\left(m x^{4}+n x^{2}\right) / 2$. We may replace 50 by 49 if $D=3$, and by 48 if $D=6$. The theorem holds if $I \geqq G+6 A$, where $A$ is large enough to satisfy (12), $\left(9^{\prime}\right)$ and $\left(10^{\prime}\right)$, while $G$ is the largest of $2 f(1), f(1)+f(2)$ and $f(3)$ if $D=1 ; G=2 f(1)$ if $D=2 ; G=f(1)$ if $D=3 ; G=0$ if $D=6$. An equivalent statement is that every integer $\geqq(G+6 A) / D$ is a sum of $t$ values of $\left(m x^{4}+n x^{2}\right) /(2 D)$, which is always integral, where $t=50$ if $D=1$ or $2, t=49$ if $D=3, t=48$ if $D=6$. 
6. For the case of polygonal numbers of order $m+2$, we have $n=2-m$. Then $0<P<4 \cdot 3^{1 / 2} m(2 m-1)$ if $m \geqq 2$, and (12) is seen to hold if $A \geqq 28 m^{3}$ (first proved by Legendre). Also $\left(9^{\prime}\right)$ holds if $A \geqq 5 m / 6$, and (10') if $A \geqq 2 m^{3}$. But (12) holds for smaller values of $A$. For example, if $m=3$, (12) holds if $A \geqq 478$, whereas $28 m^{3}=756$. By the writer's* table of sums of four polygonal numbers, the case $m=3$ shows that every integer $\leqq 480$, except the six in Theorem 5 , is a sum of four pentagonal numbers.

Theorem 5. Every integer except 9, 21,31,43, 55, and 89, is a sum of four pentagonal numbers $\left(3 x^{2}-x\right) / 2$. Hence every integer is a sum of five, one of which is 0 or 1.

Corollary 1. Except when $N$ is one of those six numbers, $24 N+4$ is a sum of four squares of integers $6 x-1$ with $x \geqq 0$.

COROLLARY 2. If $v$ is a fixed one of the numbers 2-4, 6-9, every positive integer is a sum of four integers each of which is $v$ or pentagonal.

For, each of the six exceptions in Theorem 5 is such a sum.

7. In this section we prove two lemmas.

LEMMA 1. Let $f(z)$ be an integer $\geqq 0$ for every integer $z \geqq 0$. Let $g(x)$ denote the greatest integer $\leqq f(x+1) / f(x)$. Then every positive integer $I<f(x+1)$ exceeds a sum of at most $g(x)+g(x-1)+\cdots+g(1)$ values of $f(z)$ by an integer which is $\geqq 0$ and $<f(1)$.

For, $I=C(x) f(x)+r(x)$, where $C(x) \leqq g(x), 0 \leqq r(x)<f(x), C$ and $r$ being integers. Thus every $r(x)$ is expressible as $C(x-1) f(x-1)+r(x-1)$. Repetitions show that

$$
I=C(x) f(x)+C(x-1) f(x-1)+\cdots+C(1) f(1)+u,
$$

where $0 \leqq u<f(1)$.

Lemma 2. Define $f(z)$ and $g=g(2)$ as in Lemma 1. Let $f(0)=0, f(1)=1$, and $f(z+1)>f(z)$. Then $I=g f(2)-1$ is a sum of $g-2+f(2)$, but not fewer, values of $f(z)$ for integers $z \geqq 0$.

Since $g f(2) \leqq f(3), I<f(3)$ and the only decompositions of $I$ are of the form $r f(2)+s$ with $r<g$. When $r=g-1$, then $s=f(2)-1$ and we see that $I$ is a sum of $g-1+f(2)-1=v$ values of $f(z)$. When $r=g-k, k \geqq 2$, then $s=k f(2)-1$ and the decomposition involves $v+(k-1)[f(2)-1]>v$ values of $f(z)$.

* Bulletin of the American Mathematical Society, vol. 33 (1927), p. 718. The present result was also proved directly. 
It is a reasonable conjecture that every positive integer is a sum of $I$ values of $f(z)$.

8. We now prove three theorems.

THEOREM 6. Every positive integer is a sum of 50 values of $f(x)=\left(3 x^{4}-x^{2}\right) / 2$.

Here $f(1)=1, f(2)=22, f(3)=117, f(4)=376, f(5)=925$. Since $m=3$, $n=-1$, (5) is pentagonal, whence $A=89$ by Theorem 5. In Theorem 4, $G=f(3)$, whence every integer $\geqq G+6 A=651$ is a sum of 50 values of $f(x)$. By Lemma 1 every integer $<f(4)$ is a sum of $3+5+22$ values of $f(z)$. Hence everyone $\leqq 2 f(4)=752$ is a sum of 31 values.

THEOREM 7. Every positive integer is a sum of 50 values of $f(x)=\left(5 x^{4}\right.$ $\left.-3 x^{2}\right) / 2$.

Here $m=5, n=-3$ and (12) holds if $A \geqq 2613$ (whereas Legendre's limit is 3500), and then $\left(9^{\prime}\right)$ and $\left(10^{\prime}\right)$ are satisfied. The successive values of $f(x)$ are $1,34,189,616,1525,3186,5929,10144,16281$; those of $g(x)$ are $34,5,3,2$, $2,1,1,1$. By Lemma 1 , every integer $\leqq f(9)$ is a sum of 49 values of $f(x)$. In Theorem $4, G=f(3)$, whence every integer $\geqq 189+6(2613)=15867$ is a sum of 50 values.

THEOREM 8. If every positive integer is a sum of not more than 50 values of $f(x)=\left(m x^{4}+n x^{2}\right) / 2$, then $f(x)$ is the quartic in Theorems 1,6 , or 7 , or else is

$$
f(x)=\left(7 x^{4}-5 x^{2}\right) / 2 .
$$

Since $f(y)$ shall represent 1 for an integer $y \geqq 0, y^{2}$ must divide 2 . Hence $y=1$ and $(m+n) / 2=1$. Employ (13). Then $N=1-M$,

$$
f(x)=\left(\frac{1}{2}+M\right) x^{4}+\left(\frac{1}{2}-M\right) x^{2}, M \geqq 0 .
$$

If $M=0,1$, or 2 , we have the functions in Theorems 1,6 , or 7 . Hence let $M \geqq 3$. Since $f(1)=1, f(2)=10+12 M, f(3)=45+72 M$, the number $9+12 M$ is not a sum of fewer than $9+12 M$ values of $f(x)$. But $9+12 M \leqq 50$ only if $M \leqq 3$. Thus $M=3$ and we get (14). Then $f(1)=1, f(2)=46, f(3)=267$. By Lemma $2,229=4 \cdot 46+45$ is a sum of 49 , but not fewer, values of $f(x)$. It was readily verified that 49 values suffice to $f(4)=856$, but no further examination has been made.

One of my students is treating the many universal theorems obtained when $D=2,3$ or 6 .

9. Finally, we take $m=2 u, n=2 v, u$ and $v$ relatively prime. We shall choose $A$ so large that there are $m / 2$ consecutive positive odd integers $t_{k}$ between $L$ and $G$ in (9). If $2 A-n t_{i} \equiv 2 A-n t_{j}(\bmod m)$, then $t_{i} \equiv t_{j}(\bmod m / 2=u)$.

First, let $u$ be odd. Then $t_{i} \equiv t_{j}(\bmod m=2 u)$. But the difference between 
$t_{i}$ and $t_{j}$ is numerically $<m$. Hence they are equal. Since the $m / 2$ even integers $2 A-n t_{i}$ are incongruent modulo $m$, one of them is congruent to zero. Thus

$$
k=\frac{2 A-n t}{m}=\frac{A-v t}{u}
$$

is an integer. It is odd* if $A$ is odd and $v$ is even.

Second, let $u$ be even, $v$ and $A$ both odd. Use only the first $m / 4$ of our integers $t_{k}$. The difference between any two of them is numerically $\leqq 2(m / 4$ $-1)<m / 2$. Hence we have $m / 4$ multiples $2 A-n t_{i}$ of 4 which are incongruent modulo $m$. Thus one of them is $\equiv 0$. We employ the resulting $k$ (corresponding to $t$ ) if $k$ is odd. But if $k$ is even, $k^{\prime}$ (corresponding to $t^{\prime}=t+m / 2$ ) is $k-n / 2$, which is odd, and now we have used the last $m / 4$ of our $t_{k}$.

This amplification of Maillet's argument shows that, if $u$ and $v$ are relatively prime, and if $u+v$ is odd', every sufficiently large odd integer $A$ is a sum of four values of $q(x)$ in (3).

There will be $m / 2$ odd integers between $L$ and $G$ if $G-L>m$, and hence by (9) if (10) holds with $T$ replaced by

$$
T_{1}=n-2 m+2 m^{2} \text {. }
$$

Then (11) and (12) hold with $T$ and $P$ replaced by $T_{1}$ and $P_{1}$, where

$$
P_{1}{ }^{2}=16 m^{2}\left(1+3 n-6 m+3 m^{2}\right) .
$$

Theorem 9. Let $u$ and $v$ be relatively prime, and $u+v$ be odd. Let $P_{1} \geqq 0$, $T_{1} \geqq 0$. Then $A$ is a sum of four values of $q(x)$ in (3) for integers $x \geqq 0$ if $A \geqq \frac{1}{2} m$, $A$ is odd and large enough to satisfy $\left(9^{\prime}\right),\left(10^{\prime}\right)$ and (12) with subscripts 1 on $T$ and $P$, and with the abbreviations (11), (15), (16).

10. Then (4) used four times shows that $6 A=6(2 N+1)$ is a sum of 48 values of $f(x)$ in (3). The g.c.d. of $f(1)=u+v$ and 12 is $\delta=1$ or 3. If $g$ is any positive multiple of $\delta, f(1) y+12 z=g$ is solvable in integers $y, z$ with $0 \leqq y \leqq 11$. As shown by elimination of $g, 6 A+g$ is a sum of $48+y \leqq 59$ values of $f(x)$. Hence every large multiple of $\delta$ is a sum of 59 values of $f(x)$.

But we may reduce Maillet's 59 to 51 . First, let $u+v$ be prime to 3 (as well as to 2). We employ

$$
f(0)=0, f(1)=u+v, f(2) \equiv 4(u+v), f(3) \equiv 9(u+v) \quad(\bmod 12) .
$$

Their sums by two are congruent to the products of $u+v$ by $0-2,4-6,8-10$,

* Also if $A$ is even and $v$ odd. But the resulting theorem is a mere corollary to Theorem 3, with $m$, $n$ replaced by $u, v$, as seen by doubling the numbers and function. 
whence their sums by three give a complete set of residues modulo 12 . Next, if $u+v$ is divisible by 3 , we see that $f(0), f(1), 2 f(1), 3 f(1)$ are congruent modulo 12 to $0,3,6,9$ in some order.

THEOREM 10. Let $u, v$ be relatively prime, $u>0$, and $u+v$ be odd. The g.c.d. of 12 and $u+v$ is $\delta=1$ or 3 . If $\delta=1$, let $G$ be the greatest of $3 f(1), f(1)+f(2)$, $2 f(2), f(3)+2 f(1)$ and $2 f(3)+f(1)$. But if $\delta=3$, let $G=3 f(1)$. Let $A$ be odd and large enough to satisfy $\left(9^{\prime}\right),\left(10^{\prime}\right)$ and $(12)$ in the sense of Theorem 9 . Then every integer $\geqq(6 A+G) / \delta$ is a sum of 51 values of $Q(x)=\left(u x^{4}+v x^{2}\right) / \delta$.

11. We now prove

THEOREM 11. Employ the assumptions and notations of Theorem 10. If every positive integer is a sum of 51 values of $Q(x)$, then $u+v=\delta, u \leqq 4 \delta$. Either $\delta=1$ and $u \leqq 3$, or $\delta=3$ and $u$ is one of the integers* $1,2,4,5,7,8,10,11$ prime to 3. Conversely, every integer is a sum of 51 values at least when $Q(x)=2 x^{4}-x^{2}$ or $\left(x^{4}+2 x^{2}\right) / 3$.

Since $1=Q(y)$ for an integer $y>0, y^{2}$ divides $\delta=1$ or 3 . Hence $y=1$, $u+v=\delta$. Also $Q(1)=1, Q(2)=(12 u+4 \delta) / \delta, Q(3)>Q(2)$. Hence the only decomposition of $Q(2)-1$ into a sum of values of $Q(x)$ is that in which each $x=1$. Thus $Q(2)-1 \leqq 51$, whence $u \leqq 4 \delta$. But if $\delta=1, u=4$, then $Q(2)=52$, $Q(3)=5 \times 52+37$, and Lemma 2 with $g(2)=5$ shows that 259 is a sum of 55 , but not fewer, values.

If $u=2, v=-1$, we find by Theorem 9 that if $A$ is odd and $\geqq 195, A$ is a sum of four values of $q(x)=2 x^{2}-x$. The successive values of $Q(x)=2 x^{4}-x^{2}$ are $1,28,153,496,1225,2556$, whence those of $g(x)$ in Lemma 1 are 28, 5, 3, 2 , 2, whence 40 values suffice to $F(6)=2556$, which exceeds $6 A+G=6 \times 195$ $+307=1477$.

Let $u=1, v=2$. By Theorem 9 , we find that every odd integer $A \geqq 55$ is a sum of four values of $q(x)=x^{2}+2 x$. The successive values of $Q(x)=\left(x^{4}+2 x^{2}\right) / 3$ are $1,8,33,96,225$. Those of $g(x)$ in Lemma 1 are $8,4,2,2$, whence every positive integer $\angle Q(5)=225$ is a sum of 16 values of $Q(x)$. But in Theorem 10, $G=67$, whence all integers $\geqq 133 \geqq(67+330) / 3$ are sums of 51 values.

It is readily verified that $1,2,4,5,7,10,13,20,25,28,37,52$ are the only integers $\leqq 56$ which are not sums of four values of $x^{2}+2 x$. Hence every odd integer $J$ except $1,5,7,13,25,37$ is a sum of four values with $x \geqq 0$. Write $y=x+1$. Then $J+4$ is a sum of four values of $y^{2}, y \geqq 1$.

CoRollary. Every positive odd integer except 5, 9, 11, 17, 29, 41 is a sum of four squares each $\neq 0$.

There is no such result in the literature.

* If $u=11$, Lemma 2 shows that 239 is a sum of 51 , but not fewer, values. 
PART II. WARING THEOREMS FOR CUBIC FUNCTIONS

12. In these Transactions, 1934 , pp. 1-12, I discussed

$$
f(x)=x+\frac{1}{6} \epsilon\left(x^{3}-x\right) \quad(\epsilon \text { an integer }>0)
$$

which is an integer $\geqq 0$ for every integer $x \geqq 0$, while $f(x)=1$. For $\epsilon$ prime to 3 , I found positive integers $C$ and $\nu$ such that every integer $\geqq C \cdot 3^{3 v}$ is a sum of nine values of $f(x)$ for integers $x \geqq 0$.

Call $f(x)$ universal if every positive integer is a sum of nine values of $f(x)$ for integers $x \geqq 0$. Since $f(2)=2+\epsilon, f(3)=3+4 \epsilon, 10$ is not a sum of 9 values if $\epsilon \geqq 9$. Lemma 2 shows that if $\epsilon=8,29$ is not a sum of fewer than 11 values; while if $\epsilon=7,26$ is not a sum of fewer than 10 values.

If $\epsilon=6, f(x)=x^{3}$ is known to be universal. If $\epsilon=2, f(x)$ is universal (loc. cit.). That $f(x)$ is universal if $\epsilon=3$ was proved by Frances Baker in her Chicago dissertation (photo-printed). It has since been verified that $f(x)$ is universal* if $\epsilon=1,4,5$.

THEOREM 12. Every positive integer is a sum of nine values of (17) for integers $x \geqq 0$ if and only if $\epsilon=1, \cdots, 6$.

13. The result quoted at the beginning of $\$ 12$ is a special case of

THEOREM 13. If $\epsilon$ and $\sigma$ are relatively $\dagger$ prime positive integers and if $\epsilon$ is prime to 3 , there exist positive integers $C$ and $\nu$ such that every integer $\geqq C \cdot 3^{3 v}$ is a sum of nine values of

$$
f(x)=\sigma x+\frac{1}{6} \epsilon\left(x^{3}-x\right) .
$$

LEMмa 3. Let $s$ denote the least integer $\geqq 0$ for which $3^{8} \geqq \sigma$. If $n \geqq s+1$ and $m<\epsilon \cdot 3^{n}$, then $f(3 m)<\gamma \cdot 3^{3 n}$, where $\gamma=\left(9 \epsilon^{4}+1\right) / 2$. If $\epsilon=1$ and $\sigma \leqq 3^{15} / 2$, it suffices to take $n \geqq 8$.

The condition $f\left(3 \epsilon \cdot 3^{n}\right) \leqq \gamma \cdot 3^{3 n}$ is equivalent to

$$
6 \sigma \epsilon-\epsilon^{2} \leqq 3^{2 n}, 9 \sigma^{2} \leqq(\epsilon-3 \sigma)^{2}+3^{2 n},
$$

which holds if $\sigma \leqq 3^{n-1}$ and hence if $n-1 \geqq s$.

The proof of Theorem 13 differs only in minor details from that for the case $\sigma=1$ in these Transactions, 1934, pp. 3-12, a formula there numbered $(j)$

* If $\epsilon=1$, there is no gap $\geqq 2$ in a table of sums $\leqq 2000$ of four values, whence all positive integers $\leqq 2000$ are sums of five. If $\epsilon=4$, all positive integers $I \leqq 2000$ except $17,35,55,61,73,79,200,206$, 213,225 are sums of six values, whence every $I$ is a sum of seven. If $\epsilon=5$, the exceptions to sums by seven are 20 and 360 .

$\dagger$ If they had a common factor $g>1, g$ would divide every number represented by $f(x)$ and hence divide any sum of its values. 
being now cited as $[j]$. In [10] replace the term $3 r$ by $3 \sigma r$. In the identity below [12] replace the term $2 l$ by $2 \sigma l$. In T, [13], [16] and [17], replace the term 6 by $\sigma \sigma$. In [15] and the identity above it, replace the term 1 by $\sigma$. In [20] and $S_{i}$ above it, and in [29], replace the term -6 by $-6 \sigma$, and the first term $\mp 1$ by $\mp \sigma . \operatorname{In} \beta_{i}$ in [26] replace the term 6 by $6 \sigma$.

When $\epsilon=1$, the three inequalities [26] are satisfied if $b_{1}=5, b_{2}=7, b_{3}=11$, $C=171, n \geqq 8, \sigma \leqq 5 \cdot 3^{15} / 2$. We see that $\$ 5$ holds, with the term $-6 b_{i}$ replaced by $-6 \sigma b_{i}$ in [30]. Hence every integer is a sum of nine values of (18) if $\epsilon=1, \sigma \leqq 3^{15} / 2, \nu=8, C=171$, and also for larger values of $\sigma$ when $\nu$ is increased.

The computations of $b_{1}, b_{2}, b_{3}, n, C$ depended only on inequalities [26], the only present change in which occurs in $\beta_{i}$. Hence by choice of $n$ as a function of $\sigma$, the former values* of the $b_{i}$ and $C$ apply also here.

Near the bottom of page 8 , loc. cit., we now have $\Delta \equiv 3 r \sigma, E \equiv 3 \sigma y_{i}(\bmod \epsilon)$. Since $\epsilon$ is now prime to 6 and $\sigma$, we can choose integers $y_{i}$ so that

$$
M_{i}^{\prime}-3 \sigma y_{i}-6 \sigma-3 \sigma b_{i} \equiv 0(\bmod \epsilon), 0 \leqq y_{i}<\epsilon .
$$

Since $B_{i} \equiv 3 \sigma b_{i}(\bmod \epsilon)$, we see as on page 9 that [16] determines $Q_{i}$ as an integer $\equiv 1(\bmod 4)$. This proves Theorem 13 when $\epsilon$ is prime to 6 .

In its proof $(\$ 7)$ when $\epsilon$ is even, we have merely to multiply the terms $\pm 3 b_{i}$ and -6 by $\sigma$.

14. We next prove Theorem 13 when $\epsilon=3 a, \sigma \neq \equiv 2 a(\bmod 3)$. Then

$$
D=f(z+r)-f(z)=\sigma r+\frac{1}{2} a\left(3 z^{2} r+3 z r^{2}+r^{3}-r\right)
$$

is divisible by $3^{k}$ if and only if $r$ is. Hence there exists an integer $m$ such that any given integer is congruent to $f(m)$ modulo $3^{k}$. A slight modification of the proof of Lemma 3 yields

LEмma 4. Let $s$ be the least integer $\geqq 0$ for which $3^{\circ} \geqq \sigma$. If $n \geqq s-1$ and $0 \leqq m<a \cdot 3^{n+1}$, then for (18) with $\epsilon=3 a, f(m)<\gamma \cdot 3^{3 n}$, where $\gamma=27\left(a^{4}+1\right) / 2$.

We again employ the formulas in these Transactions with factors $\sigma$ inserted at the places mentioned in $\$ 13$, and with $f(3 m)$ replaced by $f(m)$. The essential point is that $Q_{i}$ is an integer. To prove this, apply the result above Lemma 4 with $k=n+1$. Hence if $s_{i}$ is any given integer,

$$
s_{i}=f\left(t_{i}\right)+3^{n+1} u_{i} \quad\left(0 \leqq t_{i}<3^{n+1}\right) .
$$

In $D$ take $z=t_{i}, r=3^{n+1} y_{i}$, where $y_{i}$ is an arbitrary integer. Then $D=3^{n+1} E$,

\footnotetext{
* Just as we now take $C=171$ instead of the former $C=168$ when $\epsilon=1$, so also for any $\epsilon$ a lower value of $n$ may be secured by increasing the old $C$ somewhat.
} 
where $E$ is an integer, and $E \equiv \sigma y_{i}(\bmod a)$. Denote $u_{i}-E$ by $q_{i}$ and $t_{i}+3^{n+1} y_{i}$ by $m_{i}$. Then

$$
f\left(m_{i}\right)-f\left(t_{i}\right)=D=3^{n+1} E, s_{i}=f\left(m_{i}\right)+3^{n+1} q_{i} .
$$

Thus $M_{i}=3 q_{i}$ is the number used in the general theory. Since $\sigma$ is prime to $a$, there is a unique integer $y_{i}$ such that

$$
u_{i}-\sigma y_{i}-2 \sigma-b_{i} \sigma \equiv 0(\bmod a), 0 \leqq y_{i}<a .
$$

Since $N_{i} \equiv M_{i}, B_{i} \equiv 3 b_{i} \sigma(\bmod \epsilon)$, we see that $N_{i}-6 \sigma-B_{i} \equiv 0(\bmod \epsilon)$, so that $Q_{i}$ is an integer. As in these Transactions, vol. 36 , page 10, we may take $Q_{i} \equiv 1(\bmod 4)$.

We find the following values of $b_{i}, C$. Those in II and III were obtained by G. C. Webber.

I. $a=3 p+2 . b_{1}=14 p+7, b_{2}=20 p+11, b_{3}=30 p+15$. If $p=0,3076 \leqq C$ $\leqq 3089$. If $p \geqq 1, C$ is between

$$
\frac{1}{8} I_{3}=30493 \frac{1}{2} p^{4}+66136 \frac{1}{2} p^{3}+53480 \frac{1}{4} p^{2}+19130 \frac{3}{8} p+2556 \frac{7}{12}
$$

and $^{*} A+B, A=36000 p^{4}, B=80262 p^{3}+64827 p^{2}+231155 \frac{1}{2} p+3089$.

II. $a=3 p+1 . \quad b_{1}=14 p+5, p_{2}=20 p+7, p_{3}=30 p+11$. If $p=0,503 \leqq C$ $\leqq 514$. If $p \geqq 1, C$ is between

$$
\begin{aligned}
& \frac{1}{8} I_{3}=30496 \frac{1}{2} p^{4}+43699 \frac{1}{2} p^{3}+23469 \frac{3}{4} p^{2}+5601 \frac{1}{8} p+502 \frac{19}{24}, \\
& \frac{1}{3} S_{2}=36000 p^{4}+49800 p^{3}+25830 p^{2}+5954 \frac{1}{2} p+514 \frac{5}{6} .
\end{aligned}
$$

III. $a=3 p$. If $p=1, b_{1}=9, b_{2}=13, b_{3}=19,8507 \leqq C \leqq 9844$. If $p \geqq 2, C$ is between

$$
\begin{aligned}
& \frac{1}{8} I_{3}=30496 \frac{1}{2} p^{4}+57712 \frac{1}{2} p^{3}+36551 \frac{1}{4} p^{2}+7718 \frac{3}{8} p+1 \frac{1}{2}, \\
& \frac{1}{3} S_{2}=36000 p^{4}+70200 p^{3}+45630 p^{2}+9886 \frac{1}{2} p .
\end{aligned}
$$

15. Hilbert $\dagger$ proved that a polynomial in $x$ of degree $d$ with rational coefficients has an integral value for every integer $x$ exceeding a fixed limit if and only if it is a linear function with integral coefficients of the binomial coeffcients $\left(\begin{array}{l}x \\ s\end{array}\right)$ for $s=1, \cdots, d$. Replacing $x$ by $x+1$, we see that every such cubic polynomial is the sum of

$$
P(x)=A\left(x^{3}-x\right) / 6+B\left(x^{2}-x\right) / 2+C x \quad(A, B, C \text { integers })
$$

and an integer, which we may take to be zero in a Waring problem. As in the footnote to Theorem 13, we may assume

$$
A, B, C \text { have no common factor; } A>0 \text {. }
$$

* $S_{1}=37044 p^{4}+B$, while $A$ is the leading term of $\frac{1}{3} S_{2}$.

$\dagger$ Mathematische Annalen, vol. 36 (1890), p. 511. 
R. D. James* has proved that every integer exceeding a certain $L(A, B, C)$ is a sum of nine values of $P(x)$ if $A \not \equiv 4 C(\bmod 8)$. This function $L$ was not determined, but is excessively large. Unlike our results in \$\$12-14, James's result is essentially only an asymptotic theorem and yields only asymptotic results for sextics (Part III).

Part III. WARING THEOREMS FOR POLYNOMIALS OF DEGREe 6

16. We list some identities of degree 4 needed later. Write

$$
r=a^{4}+b^{4}+c^{4}+d^{4}, t=\sum_{6} a^{2} b^{2}, r+2 t=s^{2},
$$

where $s=a^{2}+b^{2}+c^{2}+d^{2}$. Then

$$
\sum_{12} \sum_{4}(A a \pm B b \pm B c)^{4}+12 A^{2}\left(4 B^{2}-A^{2}\right) r \equiv 24\left(2 A^{2} B^{2}+B^{4}\right) s^{2} .
$$

For $A=2 B$ or $A=B$, this becomes

$$
\sum_{48}(2 a \pm b \pm c)^{4} \equiv 216 s^{2}, \sum_{16}(a \pm b \pm c)^{4}+12 r \equiv 24 s^{2} .
$$

For $A=0,(26)$ becomes $\left(1_{2}\right)$. Next

$$
\sum_{24}(A a \pm B b)^{4}-6\left(A^{2}-B^{2}\right)^{2} r \equiv 12 A^{2} B^{2} s^{2},
$$

where the coefficient of $r$ is $\geqq 0$ only when $A^{2}=B^{2}$, and then (28) becomes (12). Again,

$$
\begin{aligned}
& \sum_{8}(a \pm b \pm c \pm d)^{4}+\sum_{4}(2 a)^{4} \equiv 24 s^{2} \\
& \sum_{32}(2 a \pm b \pm c \pm d)^{4}+88 r \equiv 240 s^{2} .
\end{aligned}
$$

Except for $\left(1_{2}\right)$ and (29), every such identity involves more than 32 fourth powers.

17. We employ symmetric functions of $a, b, c, d$ of degree 6:

$$
\begin{gathered}
i=\sum_{1} a^{6}, j=\sum_{12} a^{4} b^{2}, k=\sum_{4} a^{2} b^{2} c^{2}, \\
\sum_{8}(a \pm b \pm c \pm d)^{6} \equiv 8 i+8 \cdot 15 j+8 \cdot 6 \cdot 15 k, \\
\sum_{24}(g a \pm h b)^{6} \equiv 6\left(g^{6}+h^{6}\right) i+30 w j, w=g^{4} h^{2}+g^{2} h^{4} .
\end{gathered}
$$

* American Journal of Mathematics, vol. 56 (1934), pp. 303-315. 
Every linear combination of $i,(32)$ and (33) which is identical with a multiple of $s^{3}=i+3 j+6 k$ is a multiple of

$$
w(32)+8(33)+M \sum(2 a)^{6} \equiv 120 w s^{3}, 4 M=7 w-3 g^{6}-3 h^{6} .
$$

In the left member of (34) replace each exponent 6 by 4 . By (28) and (29), the resulting function becomes

$$
\left(24 w+8 \cdot 12 g^{2} h^{2}\right) s^{2}+6 \cdot 8\left(g^{2}-h^{2}\right)^{2} r+(M-w) \sum(2 a)^{4} .
$$

The sum of the last two parts will be zero if

$$
4\left(g^{2}-h^{2}\right)^{2}+w-g^{6}-h^{6} \equiv\left(g^{2}-h^{2}\right)^{2}\left(4-g^{2}-h^{2}\right)=0 .
$$

But if the last factor is zero when $g$ and $h$ are integers, either $g^{2}=4, h=0$, or vice versa, whence $M$ is negative. For a Waring problem, $M \geqq 0$. Hence $g^{2}=h^{2}$ and (34) becomes the double of

$$
g^{6}(32)+8 \sum_{12}(g a \pm g b)^{6}+g^{6} \sum(2 a)^{6} \equiv 120 g^{6} s^{3},
$$

while the like sum of fourth powers is equal to $\left(24 g^{6}+48 g^{4}\right) s^{2}$.

When $g=1$, (35) is Kempner's* identity

$$
\sum_{8}(a \pm b \pm c+d)^{6}+8 \sum_{12}(a \pm b)^{6}+\sum_{4}(2 a)^{6} \equiv 120 s^{3} .
$$

The corresponding sum $\dagger$ of fourth powers was seen to be $72 s^{2}$. That of squares is $60 s$. For arbitrary $u, v, w$, write

$$
f(x)=u x^{6}+v x^{4}+w x^{2}, q(x)=120 u x^{3}+72 v x^{2}+60 w x .
$$

Hence

$$
q(s) \equiv \sum_{8} f(a \pm b \pm c \pm d)^{6}+8 \sum_{12} f(a \pm b)+\sum_{4} f(2 a) .
$$

If we take $d=0$ in (38), we see that $q\left(a^{2}+b^{2}+c^{2}\right)$ is a sum of 107 values of $f(x)$. If we take $d=c$, and note that $f(c-d)$ becomes $f(0)=0$, we see that $q\left(a^{2}+b^{2}+2 c^{2}\right)$ is a sum of 100 values of $f(x)$. Every positive integer not of the form $h=4^{k}(16 m+14)$ is represented by $a^{2}+b^{2}+2 c^{2}$. But $h$ is not of the form $4^{k}(8 n+7)$ of the only positive integers not represented by $a^{2}+b^{2}+c^{2}$. This proves

Theorem 14. If $j$ is any positive integer, $q(j)$ is a sum of 107 values of $f(x)$ for integers $x$.

18. We identify $q(x)$ in (37) with the product of (23) by a rational con-

* Dissertation, Göttingen, 1912. Extract in Mathematische Annalen, vol. 72 (1912), p. 396.

$\dagger$ Directly by adding (29) to the product of $\left(1_{2}\right)$ by 8 . 
stant $k$, and insert the resulting values of $u, v, w$ into $f(x)$. The latter now has the denominator 720 . Hence we write $k=720 \mathrm{~N} / D$, where the integers $N$ and $D$ are relatively prime. We get

$$
\begin{aligned}
& q(x)=720 \frac{N}{D} P(x), \quad f(x)=\frac{N}{D} F(x), \\
& F(x)=A x^{6}+5 B x^{4}+(12 C-2 A-6 B) x^{2} .
\end{aligned}
$$

In a Waring theorem with summands $f(x)$, the value of $f(x)$ is assumed to be integral for every integer $x$. Hence $D$ divides $F(x)$ for every integer $x$. We have

(40) $F(x)=A\left(x^{6}-x^{2}\right)+5 B\left(x^{4}-x^{2}\right)+E x^{2}, E=12 C-A-B$,

(41) $F(1)=E, F(2)=4(15 A+15 B+E), F(3)=9(80 A+40 B+E)$.

Hence $D$ divides $E, 60(A+B), 360(2 A+B)$ and therefore their combinations, $720 C, 360 A$. Since $D$ divides the products of $A, B, C$ by 720 and since 1 is a linear combination of $A, B, C$ by (24), we see that $D$ divides 720 .

By $\$ 15$, every sufficiently large integer is a sum of nine values of $P(x)$. Hence by (39), every large multiple of $N(720 / D)$ is a sum of nine values of $q(x)$. Then by Theorem 14, the same multiple is a sum of $9 \times 107$ values of $f(x)=N F(x) / D$. This statement is evidently equivalent to the case $N=1$ of it and hence to Lemma 5.

Lemma 5. Let $D$ divide all the values of $F(x)$ in (22) for integers $x$. Then $D$ divides 720. Every sufficiently large multiple of $720 / D$ is a sum of 963 values of $F(x) / D$.

\section{This implies}

LEMma 6. Let $L$ be the least positive integer such that every integer is congruent modulo $720 / D$ to a sum of $L$ values of $F(x) / D$. Then every sufficiently large integer is a sum of $L+963$ values of $F(x) / D$.

19. We seek the number corresponding to $L$ when the modulus is one of the relatively prime factors $5,9,16$ of 720 . We are obliged to go into details to obtain facts which overcome the difficulty that congruent arguments need not yield congruent values of a polynomial whose coefficients are not integers (\$21).

If $E$ is prime to 5, (41) shows that the sums by two of the values of $F(0)$, $F(1), F(2)$ are congruent modulo 5 to $0, E, 2 E, 3 E, 4 E$, whence every integer is congruent to a sum of two values of $F(x)$. But if $E$ is divisible by $5,(40)$ and $x^{5} \equiv x$ show that $F(x) \equiv 0(\bmod 5)$ for every $x$, and we employ $F(x) / 5$. 
Modulus 9. Evidently $F(9 \pm x) \equiv F(x)$. Hence all values of $F(x)$ are congruent to those with $x=0,1,2,4$. But $3(A+B) \equiv-3 E$. Hence

$$
F(2) \equiv 7 E, F(4)=16(255 A+75 B+E) \equiv 4 E \text {. }
$$

If $E$ is prime to 3, every integer is congruent to a sum of three values of $0, E$, $4 E, 7 E$ modulo 9 .

Next, let $E=3 k$, where $k$ is prime to 3 . Then $x\left(x^{2}-1\right)$ and hence also $F(x)$ is divisible by 3 for every $x$. We see that $F(x) / 3 \equiv 0$ or $k$ according as $x$ is or is not divisible by 3 . Thus every integer is congruent to a sum of two values of $F(x) / 3$. It follows also that, for all integers $x, j$,

$$
\frac{1}{3} F(x+3 j) \equiv \frac{1}{3} F(x)
$$

$(\bmod 3)$.

But if $E$ is divisible by 9 , we employ $F(x) / 9$.

20. Modulus 16 . Evidently $F(8 \pm x) \equiv F(x)$. Also, $4(A+B) \equiv-4 E$. Hence every value of $F(x)$ is congruent to one of

$$
F(0)=0, F(1)=E, F(2) \equiv 8 E, F(3) \equiv 9 E+8 B .
$$

Case $B$ even. We may drop the term $8 B$ from (43). If $E$ is odd, every integer is congruent to a sum of 7 values of $F(x)$.

Let $E=2 m$. Then $F(x)$ is always even. Also $F(x) / 2 \equiv 0$ or $m(\bmod 8)$ according as $x$ is even or odd; and hence

$$
\frac{1}{2} F(x+2 j) \equiv \frac{1}{2} F(x)(\bmod 8), \text { for all } x, j .
$$

When $m$ is odd every integer is congruent modulo 8 to a sum of 7 values of $F(x) / 2$. If $E=4 M$, where $M$ is odd, we require a sum of 3 values of the residues $0, M$ modulo 4 of $F(x) / 4$ which is always integral. If $E=8 M, M$ odd, every integer is congruent modulo 2 to one of the values $0, M$ of $F(x) / 8$, which is always integral. Finally, if $E$ is divisible by 16 , we use $F(x) / 16$, which is always integral.

Case $B$ odd. If $E$ is odd, then $F(3) \equiv E$, and we use a sum of 7 values of $F(x)$. If $E=2 m$, where $m$ is odd henceforth, the values of $F(x) / 2$ are $\equiv 0, m$, $5 m(\bmod 8)$, a sum of four of which yields every residue. Also,

$$
\frac{1}{2} F(x+8 j) \equiv \frac{1}{2} F(x)(\bmod 8), \text { for all } x, j .
$$

Let $E=4 m$. Then, $F(2 y) / 4 \equiv 0$ and

$$
\frac{1}{4} F(1) \equiv \frac{1}{4} F(7) \equiv m, \frac{1}{4} F(3) \equiv \frac{1}{4} F(5) \equiv-m \quad(\bmod 4),
$$

a sum of two of which yields every residue. Here

$$
\begin{aligned}
\frac{1}{4} F(x+4) & \equiv-\frac{1}{4} F(x)(\bmod 4), x \text { odd; } \\
\frac{1}{4} F(x+8 j) & \equiv \frac{1}{4} F(x)(\bmod 4), \text { all } x, j .
\end{aligned}
$$


Let $E=8 m$. Then $F(x) / 8 \equiv 0(\bmod 2)$ unless $x \equiv \pm 1(\bmod 8)$ and then $F(x) / 8 \equiv 1(\bmod 2)$. Here

$$
\begin{aligned}
\frac{1}{8} F(x+4) & \equiv 1+\frac{1}{8} F(x)(\bmod 2), x \text { odd; } \\
\frac{1}{8} F(x+8 j) & \equiv \frac{1}{8} F(x)(\bmod 2), \text { all } x, j .
\end{aligned}
$$

21. It remains to pass from our relatively prime moduli to the product as modulus. In case a polynomial $p(z)$ has integral coefficients, the classic method is to employ the Chinese remainder theorem and note that

$$
z \equiv a(\bmod M) \text { implies } p(z) \equiv p(a)(\bmod M) .
$$

By (42), (44) and (45), property (50) holds also for our corresponding polynomials having denominators. There remain the cases $M=4,2$ when $B$ is odd. In view of (47) and (49), we have only to apply the Chinese remainder theorem when one congruence is $z \equiv a(\bmod 8)$ instead of $z \equiv a(\bmod M=4$ or 2).

\section{We have now proved}

THEOREM 15. Let $D$ be the largest integer which divides all the values of $F(x)$ in (40). Then $D$ is the g.c.d. of 720 and E. Then in Lemma $6, L \leqq 7$, so that every large integer is a sum of $L+963 \leqq 970$ values of $F(x) / D$. We have $L=7$ if $E$ is odd, or if $B$ is even and $E=2 m$, where (as below) $m$ is odd. Next, $L=4$ if $B$ is odd and $E=2 m$. Again, $L=3$ if $B$ is even and $E=4 m$, or if $E$ is prime to 3 and divisible by 4 . Also, $L=1$ if $E$ is divisible by 360 . In all the remaining cases, $L=2$.

22. Examples. By Theorem 12 every positive integer is a sum of nine values of $P(x)=\left(x^{3}+2 x\right) / 3$, viz., (17) for $\epsilon=2$. Hence $A=2, B=0, C=1$, and $F(x)=2 x^{6}+8 x^{2}$. Here $E=10, D=10, L=7$. By Lemma 6 , every integer is congruent modulo $720 / D=72$ to a sum of seven values of $Q(x)=\left(x^{6}+4 x^{2}\right) / 5$. Since $0, \pm 1, \cdots, \pm 35,36$ form a complete set of residues modulo 72 , every integer $\geqq 7 Q(36)$ is a sum of 970 values of $Q(x)$. Successive values of $Q(x)$ are $1,16,153,832,3145,9360,23569,52480, Q(9)=106353$. In Lemma 1 , the successive values of $g(x)$ are $16,9,5,3,2,2,2, g(8)=2$, whence every integer $<Q(9)$ is a sum of 41 values of $Q(x)$. But $Q(x+1)<2 Q(x)$ if $x \geqq 9$, whence $q(x)=1$. Hence $41+27=68$ suffice to $Q(36)$, and $6+68=74$ suffice to $7 Q(36)$.

THEOREM 16. Every positive integer is a sum of 970 (integral) values of $\left(x^{6}+4 x^{2}\right) / 5$.

Next, employ Theorem 12 with $\epsilon=3$. Then $A=3, B=0, C=1, E=D=9$, $L=7$. We see that 87 suffice to $7 H(40)$, where $H(x)=\left(x^{6}+2 x^{2}\right) / 3$. Finally, if 
$\epsilon=1, A=C=1, B=0, E=11, D=1, L=7 ; g(1)=9, g(2)=7, g(3)=5, g(4)=3$, $g(x)=2$ if $x=5-8, g(x)=1$ if $x \geqq 9$.

THEOREM 17. Every positive integer $I$ is a sum of 970 values of $\left(x^{6}+2 x^{2}\right) / 3$. If $I \geqq 7 F(360), I$ is a sum of 970 values of $F(x)=x^{6}+10 x^{2}$; if $I \leqq 7 F(360), I$ exceeds a sum of 389 values of $F(x)$ by an integer which is $\geqq 0$ and $\leqq 10$.

Various similar theorems are omitted for brevity.

Part IV. WARING THEOREMS FOR CERTAIN POLYNOMIALS OF DEGREES 8 AND 10

23. Employ the notations

$$
\begin{aligned}
H(x) & =u x^{8}+v x^{4}+w x^{2}, \\
f(x) & =5040 u x^{4}+720 v x^{2}+504 w x .
\end{aligned}
$$

Then we have the identity* in $a, b, c, d, u, v, w$ :

$$
\begin{aligned}
f(s) \equiv & \sum_{48} H(2 a \pm b \pm c)+6 \sum_{4} H(2 a)+6 \sum_{8} H(a \pm b \pm c \pm d) \\
& +60 \sum_{12} H(a \pm b) .
\end{aligned}
$$

When $v=w=0$, the identity is due to A. Hurwitz. $\nmid$ When $u=w=0$, it follows from $\left(27_{1}\right),(29),\left(1_{2}\right)$.

Take $w=0$. Then $f(x)=720\left(7 u x^{4}+v x^{2}\right)$. To apply Theorem 10 , let $7 u$ and $v$ be relatively prime and $7 u+v$ be odd. Let $\delta$ be the g.c.d. of the latter and 3 . Hence every sufficiently large multiple of 720 is a sum of 51 values of $f(x)$ and hence by (51) (with 840 values of $H$ ) is a sum of $51 \times 840$ values of $H(x)$ or $H(x) / 3$ according as $\delta=1$ or 3 . A similar theorem with 51 replaced by 50 follows from Theorem 4. To apply the better Theorem 1, take $u=1 / 2, v=7 / 2$. Then $f(x)=5040\left(x^{4}+x^{2}\right) / 2$. Hence every large multiple of 5040 is a sum of $38 \times 840$ values of $\left(x^{8}+7 x^{4}\right) / 2$. Similarly by Theorem 2 , every large multiple of 30240 is a sum of $36 \times 840$ values of $\left(x^{8}-7 x^{4}\right) / 12$. We obtain Waring theorems as in Lemma 6.

24. J. Schur $\ddagger$ expressed $22680 s^{5}$ as a sum of tenth powers. Replacing each exponent 10 by 4 , we see that the sum becomes the sum of $\left(27_{1}\right)$ and the products of $(29)$ by 9 and $\left(1_{2}\right)$ by 180 , and hence is $1512 s^{2}$. But if we replace each exponent 10 by 6 or 8 we do not obtain a multiple of $s^{3}$ or $s^{4}$. Hence

* It does not hold if $H$ has a term in $x^{6}$.

$\dagger$ Mathematische Annalen, vol. 65 (1908), pp. 424-7.

$\ddagger$ Mathematische Annalen, vol. 66 (1909), p. 105; History of the Theory of Numbers, II, p. 721. 
$1512\left(15 u s^{5}+v s^{2}\right)$ is obtained from Schur's sum by replacing each $x^{10}$ by $u x^{10}+v x^{4}$. A Waring theorem for the latter may therefore be deduced from one for* $15 u x^{5}+v x^{2}$.

* Maillet, Journal de Mathématiques, (5), vol. 2 (1896), pp. 363-380, proved that every large integer is a sum of a limited number of 1 's and 192 values of any polynomial in $x$ of degree 5 which is a positive integer for all integers $x \geqq g$.

University of Chicago, Chicago, Ill. 